\title{
Immunochemical Analysis of Mutant CFTR in Lung Explants
}

\author{
Andrea van Barneveld ${ }^{a} \quad$ Isabell Zander ${ }^{b} \quad$ Rebecca Hyde $^{b} \quad$ Florian Länger $^{c}$ \\ André Simon $^{d} \quad$ Marcus Krüger $^{d} \quad$ Manfred Ballmann ${ }^{b}$ Nico Derichs ${ }^{b}$ Burkhard \\ Tümmler \\ aKlinische Forschergruppe, OE 6710, 'Klinik für Pädiatrische Pneumologie, Allergologie und \\ Neonatologie, OE 6710, 'Institut für Pathologie and 'Klinik für Herz-, Thorax-, Transplantations- und \\ Gefäßchirurgie, Medizinische Hochschule Hannover, Hannover
}

\section{Key Words}

Cystic fibrosis $\cdot$ CFTR $・$ Immunoblot $・$ Lung transplantation

\begin{abstract}
Background/Aims: Knowledge about the abundance and distribution of CFTR protein glycoforms in native lung tissue is scarce. For upcoming studies with correctors and potentiators for CFTR it is important to get more information about mutant CFTR protein biochemistry. Target for novel treatment is the most afflicted organ in cystic fibrosis (CF), the lung. Methods: Lung tissue sampled from patients with CF and non-CF donors prior to lung transplantation was examined for CFTR-immunoreactive signals by immunoblot. Quantitation of the immunoreactive signals was carried out by densitometry. Results: The complexglycosylated and mannose-rich CFTR isoforms were present in all non-CF specimens, whereas no or only the immature CFTR isoform was visible in CF samples. Whereas some complexglycosylated CFTR was often present in rectal biopsies of F508del homozygous subjects, no mature CFTR was detectable in CF lungs at the stage of terminal respiratory insufficiency. Conclusion: Immunoblot analysis of CFTR in lung tissue is feasible, but in context of the upcoming studies of CFTR correctors and potentiators rectal biopsies seem to be a more appropriate choice because of their safe and repeatable excision.
\end{abstract}

Copyright $(2012$ S. Karger AG, Basel

\section{Introduction}

Cystic fibrosis (CF) is the most common lethal disease in the Caucausian population. It is caused by mutations in the CFTR gene (OMIM 219700) which encodes a cAMP-regulated 
$\mathrm{Cl}^{-}$channel that is expressed in the apical plasma membrane of exocrine epithelia [1]. The CFTR protein pool mainly consists of a mannose-rich immature ER (band B) and a complexglycosylated mature isoform (band C) [1].

Most of our knowledge about the pathology of mutant CFTR protein resides on immunoblot analysis of cell culture [2-10]. Only few studies examined mutant CFTR in human tissue, i.e. sweat gland [11,12], intestine [12-14], nasal polyps [12] and lungs [15]. In the context of the upcoming studies on correctors and potentiators of mutant CFTR [16, 17] it is important to enlarge our knowledge about mutant CFTR protein biochemistry. Most individuals with CF carry CFTR gene mutations that give rise to defective or impaired posttranslation processing and/or trafficking $[1,18,19]$. Even in exocrine pancreassufficient patients the delivery of wild-type amounts of mutant CFTR to the apical membrane of epithelial cells is the exception rather than the rule [19].

The lung is the most afflicted organ in CF and hence is the major target for any novel treatment. To clarify the distribution and abundance of isoforms of normal and mutant CFTR we investigated snap-frozen tissue from freshly explanted non-CF and CF subjects using our protocol that had been established to examine CFTR in rectal biopsies [14].

\section{Materials and Methods}

\section{Tissues and cell lines}

Native CF lung epithelium specimens were obtained from freshly explanted CF lungs on the occasion of lung transplantation and snap frozen in liquid nitrogen. Non-CF lung epithelium specimens were analyzed from parts of non-CF donor lung explants that showed regular histology of airway epithelium and submucosal glands. Rectal biopsies were collected with a suction biopsy device from F508del homozygous CF subjects and non-CF healthy volunteers [14]. T84 cells were cultured in DMEM/Ham-F12 medium supplemented with $5 \%(\mathrm{v} / \mathrm{v})$ fetal calf serum at $37^{\circ} \mathrm{C}$ in a $5 \% \mathrm{CO}_{2}$ atmosphere.

\section{Antibodies}

The following anti-CFTR Abs were used: polyclonal R16 [14] against the N-terminus (aa 1-14, R16/34 or R66/17) and C-terminus (aa 1466-1480, R16/33 or R66/16), the crude antiserum R66, polyclonal R40 [14] against C-terminus (aa 1465-1488), mAb 596 [13] against NBD2 (aa 1204-1211) and mAb 570 [13] against R-domain (aa 731-742). All antibody lots were verified to recognize the B-and C-bands of CFTR in T84 cells.

The following anti-CLCN2 Abs were used: polyclonal anti-CLCN2 Abs C-20 and H-90 (Santa Cruz) and anti-CLCN2 mAb54664 (abcam).

\section{CFTR and CLCN2 immunochemical analyses}

The snap frozen specimens ( $\mathrm{n}=8-16)$ were homogenized at $4^{\circ} \mathrm{C}$ in the presence of $10 \mathrm{mM}$ iodoacetamide, $61 \mu \mathrm{g} / \mathrm{ml} \mathrm{PMSF}, 0.35 \mu \mathrm{g} / \mathrm{ml}$ pepstatin and antipain, $1.75 \mu \mathrm{g} / \mathrm{ml}$ leupeptin and aprotonin and $17.5 \mu \mathrm{g} / \mathrm{ml}$ soybean trypsin-inhibitor in Tris-buffer $(20 \mathrm{mM}$ Tris/ $\mathrm{HCl}, 150 \mathrm{mM} \mathrm{NaCl}, \mathrm{pH}$ 8). The lysis started by incubation with $0.03 \%$ SDS for at least $30 \mathrm{~min}$ at $4{ }^{\circ} \mathrm{C}$, followed by $1 \%(\mathrm{v} / \mathrm{v})$ Triton X-100 and $0.5 \%(\mathrm{w} /$ v) sodium deoxycholate for at least $30 \mathrm{~min}$. After centrifugation $\left(16,000 \times g, 4^{\circ} \mathrm{C}, 20 \mathrm{~min}\right)$ protein contents of the supernatant were determined [20]. Thereafter the supernatant was incubated with the specific preimmune sera and protein A agarose (PAA) for $60 \mathrm{~min}$. Immunoprecipitation (IP) was carried out overnight with the polyclonal antibodies [14] R40 (dilution 1:50 adjusted to volume), R16/33 or R66/16 (1:133, both are against the C- terminus of CFTR) and R16/34 or R66/17 (1:80 against the N-terminus of CFTR) in the presence of protein A agarose and protein $\mathrm{G}$ agarose (Santa Cruz Biotechnologies). A second IP was performed overnight with the crude antiserum R66 (1:50) or the polyclonal antibodies C-20 (1:50) and H90 (1:50). All beads were washed at $4^{\circ} \mathrm{C}$ twice with 1) PBS with $0.5 \%$ (v/v) Triton X-100 and $0.05 \%$ (w/v) sodium deoxycholate and then twice with 2) $125 \mathrm{mM}$ Tris, $500 \mathrm{mM} \mathrm{NaCl}, 10 \mathrm{mM}$ EDTA, $0.5 \%$ (v/v) Triton $\mathrm{X}-100, \mathrm{pH}$ 8. Thereafter the beads were dissolved in $50 \mu \mathrm{L} 3 \mathrm{x}$ Laemmli buffer containing dithiothreitol at $37^{\circ} \mathrm{C}$ for $3 \mathrm{~h}$, then separated by $5 \%$ SDS-PAGE (for detection of CFTR) or by 7\% SDS-PAGE (for detection of CLCN2) and blotted onto PVDF membranes. CFTR (CLCN2) immunoreactive bands were detected with mAbs 570 and 596 [13, 15] (1:500) (CLCN2 antibody ab54664 (abcam, 1:500)) in 0.2 \% I-Block (Tropix, Applied Biosystems) in 0.05\% Tween-TBS (T-TBS) and pre-adsorbed anti-mouse-IgG-HRP from donkey 
Fig. 1. Immunodetection of CFTR in rectal biopsies taken from four healthy non-CF donors (panels A - D). The first IP was performed according to the protocol described in the Materials and Methods section (lane a). The second IP of the same lysate was carried thereafter with the crude R66 antiserum (lane b). CFTR was detected on the immunoblot of SDSPAGE-separated IPs with the anti-CFTR mAbs 570 and 596 and the secondary $\mathrm{Ab}$ anti-mouse-IgG-HRP. The comparison of the immunoreactive signal in lanes $\mathrm{a}$ and $\mathrm{b}$ revealed that the first IP was almost quantitative for CFTR.

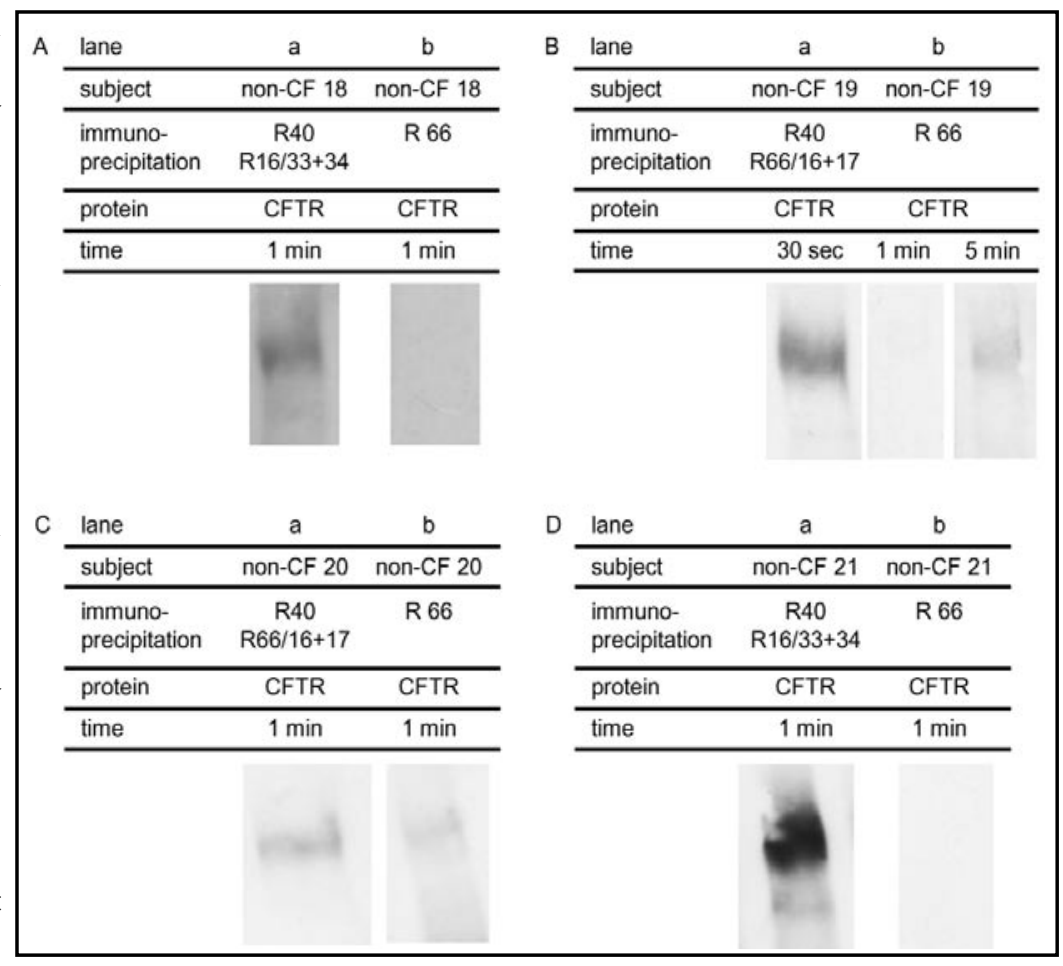

(abcam, 1:300000) (CLCN2 anti-mouse -IgG-HRP, BD, 1:300000)) in 0.2 \% I-Block in T-TBS and visualized with ECL Advance (GE Healthcare) for 20 seconds.

The CFTR/CLCN2-immunoreactive signals were quantified by densitometry of a time series of Hyperfilms ECL (GE Healthcare) exposed to ECL Advance-covered immunoblots. The density was determined on unsaturated films with exposure times between $10 \mathrm{sec}$ and up to $3 \mathrm{~min}$. For the calculation of the density the average of 5 rectangles in case of the C-band and 3 rectangles in case of the B-band were used to eliminate small inequalities of the densities within the bands. After subtraction of background intensity, all densities were normalized to one minute exposure time. Total protein was determined by the Bradford assay [20]. The density $\left(\mathrm{od} / \mathrm{mm}^{2}\right)$ of all samples was normalized by the amount of total protein. For the calculation of the ratios of $\mathrm{C}$ - to $\mathrm{B}$-band the intensities of the complete $\mathrm{C}$ - or B-bands were determined (multiplication of the area of the band with the normalized density).

\section{Results}

In our standard protocol lung specimens were lysed, the lysate was immunoprecipitated with polyclonal anti-CFTR Abs, and CFTR then visualized by highly specific and sensitive anti-CFTR mAbs on the immunoblot of the PAGE-separated IP. The advantage of an immunoprecipitation followed by a further immunoblot analysis is the enrichment of the target protein and the reduction of the background of false positive bands at the position of the target protein [13]. The specificity and sensitivity of this method depends on the availability of polyclonal and monoclonal anti-CFTR antibodies. The used high-affinity mAbs were developed in the course of recent studies by our peers on F508del homozygotes [13]. The combination of polyclonal anti-CFTR antibodies against the N- and C-terminus of CFTR and the highly sensitive and specific used mAbs led to a high sensitivity of 508del CFTR detection in human rectal biopsies [14]. The protocol has been optimized for high sensitivity. Titrations with variable amounts of the highly CFTR-expressing T84 cells revealed that only $6 \%$ of total CFTR was immunoprecipitated from the cell lysates (data not shown) whereas more than $90 \%$ of the immunodetectable CFTR was immunoprecipitated from rectal biopsies (Fig. 1). 
Fig. 2. Immunodetection of CFTR in lung and T84 cells. IPs were performed with the pre-immune sera (PPI) R40 and R16 (lanes b, f and g) or the polyclonal antiCFTR antibodies R40 and R16/33 +34 (lanes a, c-e). CFTR was detected on the immunoblot of SDS-PAGE-separated IPs with the anti-CFTR mAbs 570 and 596 and the secondary $\mathrm{Ab}$ anti-mouse-IgGHRP (lanes a-g). In lane a (T84 cells) and c (non-CF 1) a strong complex-glycosylated $\mathrm{C}$-band and a weaker mannose-rich B-band of CFTR were detectable, but not in lane b with PPI. In lane e (CF2) a weak B-band was detectable, but not in lane d (CF1) or in lanes $f$ and $g$ with PPI. Please note that the exposure time of the T84 signal in lane a was 10 times lower than that of the lung signals in lanes $b$ to $g$.

Fig. 3. A. Immunodetection of CFTR in lung, rectum and T84 cells. Lysates of T84 cells, non-CF rectal biopsies and non-CF lung epithelium specimens were immunoprecipitated with polyclonal anti-CFTR Abs. CFTR was detected on the immunoblot of SDS-PAGE-separated IPs with the anti-CFTR mAbs 570 and 596. In all lanes (a-f) the complex-glycosylated C-band and the mannose-rich B-band of CFTR were detectable. Please note the differential glycosylation pattern of band $C$ in rectum and lung. All lanes were taken from different gels. For the correct determination of the size of rectal and lung CFTR, samples from both tissues were run on the same gels. B. Immunoblot analysis of mutant CFTR in lung tissue explanted during lung transplantation. Lysates of non-CF and mutant CF lung specimens were immunoprecipitated with polyclonal anti-CFTR Abs and detected by the antiCFTR mAbs 570 and 596. In the sample from a non-CF subject (lane a) the strong complex-glycosylated C-band and a weak mannose-rich B-band of CFTR were clearly visible, but in lanes b-f only the mannoserich B-band of mutant CFTR with different mutation genotypes was clearly detectable. All lanes were taken from different gels. Blots were exposed to films for $1 \mathrm{~min}$ but samples non-CF5 (10 min), CF3 (20 min), CF5 (10 min) and T84 (10 sec).

\begin{tabular}{|c|c|c|c|c|c|c|c|}
\hline lane & a & b & c & d & e & f & g \\
\hline subject & cell & non-CF & non-CF1 & CF1 & CF2 & CF2 & CF1 \\
\hline tissue & T84 & lung & lung & lung & lung & lung & lung \\
\hline no. of biopsies & - & 16 & 16 & 16 & 16 & 16 & 16 \\
\hline protein & CFTR & PPI & CFTR & $\begin{array}{c}\text { F508del/ } \\
\text { CFTR } \\
\text { dele2,3 } \\
(21 \mathrm{~kb})\end{array}$ & $\begin{array}{l}\text { F508del/ } \\
\text { F508del }\end{array}$ & PPI & PPI \\
\hline $\begin{array}{l}\text { immuno- } \\
\text { precipitation }\end{array}$ & $\begin{array}{c}\text { R40 } \\
\text { R16/33 } \\
+34 \\
\end{array}$ & $\begin{array}{l}\text { R40 } \\
\text { R16 }\end{array}$ & $\begin{array}{c}\mathrm{R} 40 \\
\mathrm{R} 16 / 33 \\
+34 \\
\end{array}$ & $\begin{array}{c}\text { R40 } \\
\text { R16/33 } \\
+34 \\
\end{array}$ & $\begin{array}{c}\mathrm{R} 40 \\
\mathrm{R} 16 / 33 \\
+34 \\
\end{array}$ & $\begin{array}{l}\text { R40 } \\
\text { R16 }\end{array}$ & $\begin{array}{l}\text { R40 } \\
\text { R16 }\end{array}$ \\
\hline $\begin{array}{l}\text { C-band } \rightarrow \\
150 \mathrm{kDa}-\end{array}$ & & & & & & & \\
\hline B-band $\rightarrow$ & $=$ & & & & 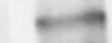 & & \\
\hline
\end{tabular}

Lung specimens were available from seventeen non-CF donors and seven individuals with CF. Samples were retrieved during lung transplantation from biopsies of non-CF donor 
Fig. 4. Determination of CFTR contents of non-CF (A) and mutant (B) CFTR in lung specimens by densitometry of immunoblots. Lysates of lung epithelium specimens were immunoprecipitated with polyclonal antiCFTR Abs. CFTR was detected on the immunoblot of SDS-PAGE-separated IPs with the anti-CFTR mAbs 570 and 596. The figure shows the blots that were selected for densitometry according to the criteria of optimal signal-to-noise ratios and a time window of linear increase of signal with exposure time. The indicated signal intensities are not normalized by the amount of protein and by time.

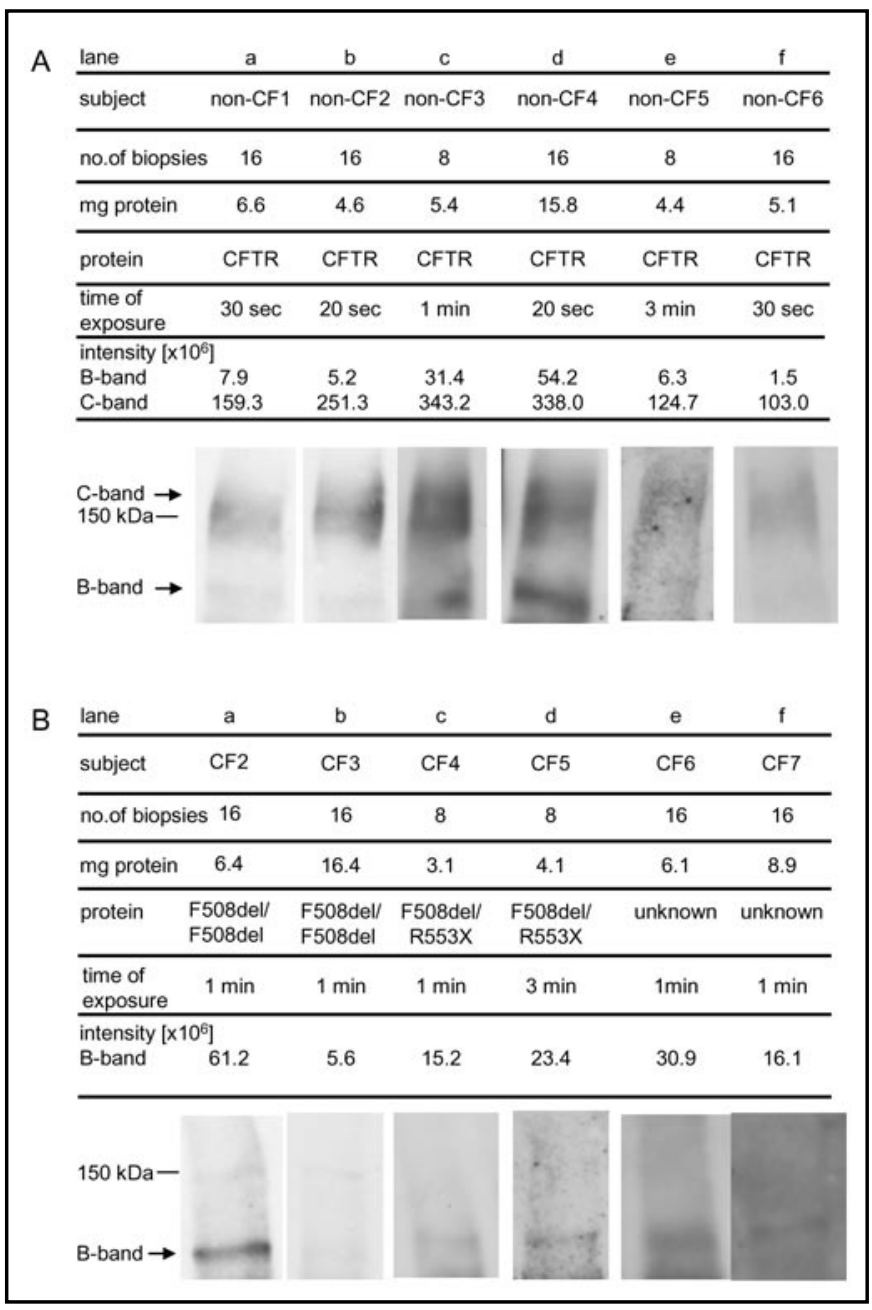

lungs that had been subject to perioperative histological examination and from the explanted CF lungs that represent end-stage lung disease. All lung specimens of non-CF subjects showed the complex-glycosylated C-band of mature CFTR and a weak, but clearly visible mannoserich B-band of the ER-isoform (Fig. 2 and 3). In CF specimens only the mannose-rich isoform of mutant CFTR or no band were detectable (Fig. 2 and 3B). No complex-glycosylated mutant CFTR was visible. The preimmune sera (PPI) of the used antibodies for immunoprecipitation did not produce any non-specific by-bands in the sensitive mol.wt. range for CFTR or mutant CFTR detection above $100 \mathrm{kDa}$ (Fig. 2, lanes b, $\mathrm{f}$ and g). Please note the differential glycosylation patterns of the C-band from T84 cells, rectal and lung tissue (Fig. $3 \mathrm{~A}$ ). The glycoforms were more disperse in lung than in rectum. In CF lung tissue the mannose-rich B-band was detectable in specimens from six of seven subjects (Fig. 2 and 3 B).

For quantitative densitometry blots with low signal intensities were used as shown in Figure 4 for lung samples. Quantitative densitometry revealed that lung tissue contained on the average about fivefold less CFTR protein than rectal epithelium (Fig. 5). The ratio of Bto $\mathrm{C}$ - band differed considerably in between non-CF lung specimens (Fig. 5). The detectable amounts of mannose-rich isoform in rectal and lung CF tissues were comparable within the same order of magnitude (Fig. 5). Rectal suction biopsies and specimens from explanted lung tissue inherently consist of variable proportions of epithelial and non-epithelial cells. To get an estimate of the amount of epithelial cells in the samples, the IPs for CFTR were combined with IPs for CLCN2, a member of the voltage-gated ClC chloride channel family that is expressed in the apical membrane of airway and intestinal epithelium [21, 22]. Hence 


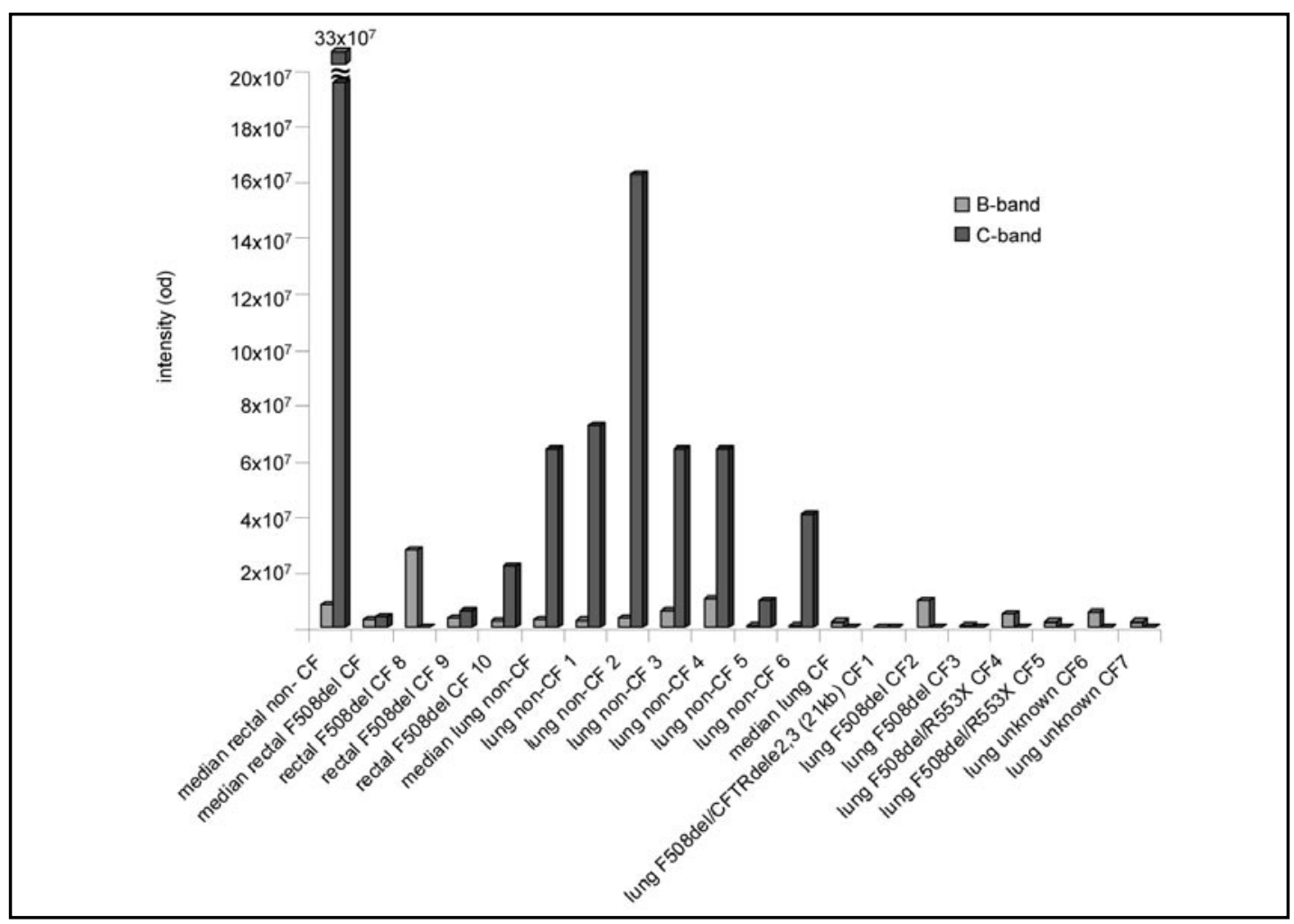

Fig. 5. Contents of CFTR B-band and C-band in immunoblots of SDS-PAGE separated anti-CFTR IPS of CF and non- CF lung specimens and rectal suction biopsies. Signal intensities of the ratios of C- to B-band were calculated from the normalized densities multiplied by the area of the bands. The normalized density indicates the immunoreactive CFTR signal on an X-ray film that had been exposed between 20 sec and up to $3 \mathrm{~min}$ to the immunoblot covered with ECL Advance. All densities of unsaturated signals were normalized to $1 \mathrm{~min}$ exposure time and $1 \mathrm{mg}$ protein. Wild-type and mutant B- and C-CFTR glycoforms were detected in highly variable amounts and $\mathrm{C} / \mathrm{B}$ ratios.

CLCN2 is a promising candidate for an epithelial marker protein. As shown in Figures 6 and 7, the proportion of epithelial cells was higher in rectal biopsies than in the examined samples of lung explants.

The majority of F508del homozygous rectal epithelia expressed low amounts of complexglycosylated CFTR [14]. In contrast no band C CFTR was detectable in all investigated CF lung specimens.

\section{Discussion}

This study investigated CFTR protein expression in CF lungs at the time of lung transplantation. No mature complex-glycosylated mutant CFTR was detectable at this stage of global respiratory insufficiency in subjects with a pancreas-insufficient CFTR genotype. The minor ER glycoform, however, was visualized with similar signal intensity on blots of $\mathrm{CF}$ and non-CF origin. In other words, the terminal stage of the remodelled and chronically inflamed CF lungs is characterized by the maintenance of the ER glycoform and the absence of the mature glycoform. If some minute band C was present in the specimen, it was lost during processing below the threshold of detection.

When applying the same immunoblot protocol we previously detected complexglycosylated CFTR in 10 of 12 rectal biopsies taken from F508del homozygotes with mild to 
Fig. 6. Immunodetection of CFTR and the apical epithelial protein CLCN2 in lung and rectum. Lysates of non-CF rectal biopsies and non-CF lung epithelium specimens were immunoprecipitated with polyclonal anti-CFTR Abs first and polyclonal antiCLCN2 Abs C-20 and H-90 (Santa Cruz) thereafter. CFTR and CLCN2 were detected on immunoblots of PAGE-separated IPs with the anti-CFTR mAbs 570 and 596 and antiCLCN2 mAb54664 (abcam), respectively.

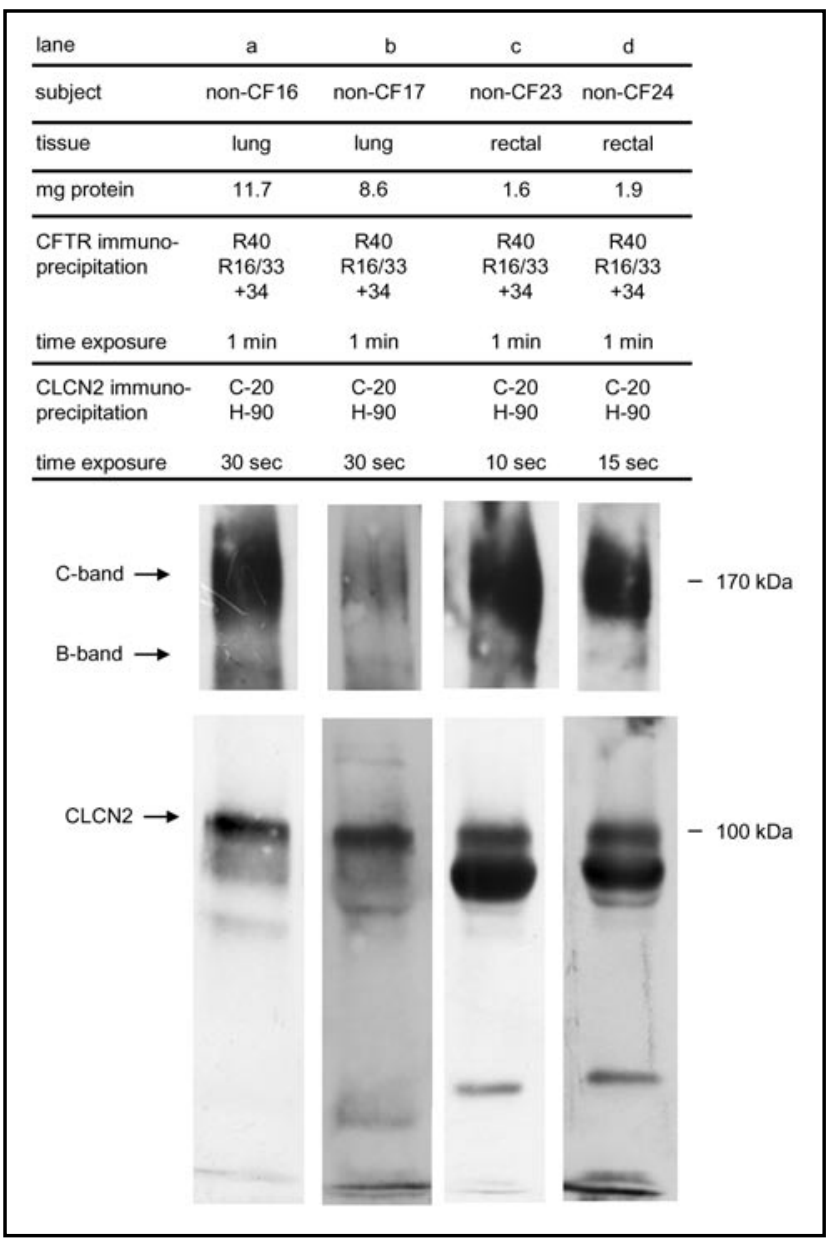

Fig. 7. Normalized immunochemical signal intensities of the contents of band C CFTR (grey columns), band B CFTR (black) and CLCN2 (white). Please note that rectal biopsies contain relatively larger amounts of epithelial cells than specimens from explanted lung tissue.

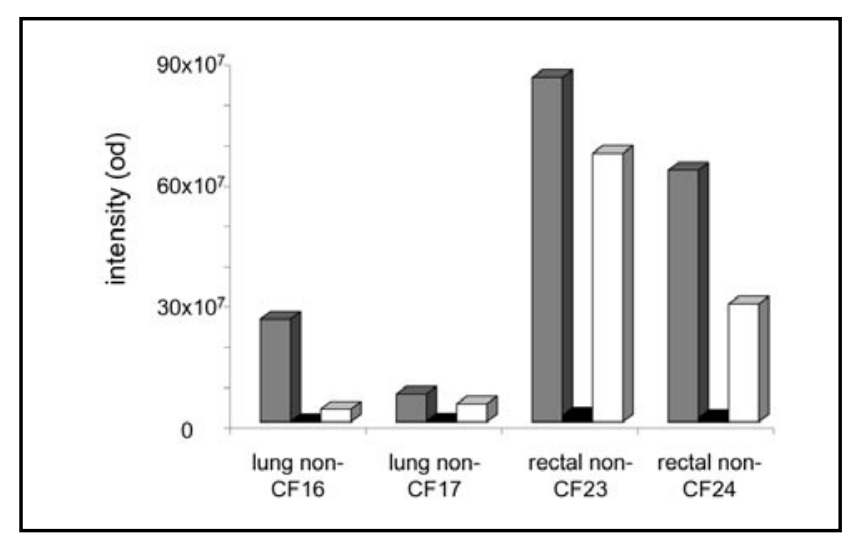

moderate CF disease (Fig. 5) [14]. Several reasons probably account for this major difference in the abundance of mutant CFTR in these two types of specimens. First, CFTR was about fivefold more strongly expressed in the distal intestine than in the majority of examined lungs (see Fig. 5; statement applies to all specimens but non-CF2). Second, the intestinal epithelium is turned over every three days, whereas airway epithelium only expands during growth and regenerates after injury. Third, CF lungs are subject to chronic inflammation, remodelling and dedifferentiation with a concomitant irreversible loss of CFTR-positive epithelial cells [1]. Thus, our immunoblot data on CFTR expression in lungs at the stage of respiratory failure do not allow any definitive statement on the CFTR protein distribution in native non-injured airways. With the exception of null mutations, some complex-glycosylated 
mutant CFTR may be present. There are reports in the literature that even processingdefective mutants such as F508del may show some CFTR-immunoreactive apical labelling in pseudostratified airway epithelium [23-25].

In conclusion, CFTR immunoblot analysis is feasible with CF lung tissue. This point is relevant in the light of the up-coming clinical trials on CFTR correctors that aim to promote proper processing and targeting of mutant CFTR to and/or to prolong residence time at the apical membrane. However, prior to any implementation of biopsies of respiratory epithelium into a clinical trial, the ex vivo analyses should be repeated with biopsies that had been taken from CF subjects with mild lung disease. CFTR protein analysis should be combined with a histological examination of the specimen to allow a proper interpretation of the immunochemical CFTR data. Moreover, the amount of CFTR should be assigned to the proportion of CFTR-positive epithelia in the examined specimen. Lung tissue is inherently heterogeneous in its composition of epithelial and non-epithelial cells, and the ratio of cell types will vary by specimen. This problem can eventually be reduced by the combination of the determination of protein contents with quantitative immunoblot densitometry of an epithelial marker protein such as CLCN2 [21, 22, 26].

By the time of writing, however, biopsies from the distal intestine seem to be a more appropriate choice to assess the action of correctors in clinical trials. As already outlined above, the rectal epithelium contains more CFTR protein than respiratory epithelium, shows a rapid turnover, is not subject to pronounced dedifferentiation and remodelling, and finally and most importantly in the context of a clinical trial, a rectal suction biopsy is safe, painless and repeatable.

\section{Acknowledgements}

The authors are grateful to John R. Riordan and his team for the provision of antiCFTR mAbs. Financial support was provided by the Deutsche Forschungsgemeinschaft (SFB $621, \mathrm{C7}$ ) and the Mukoviszidose gGmbH, the research arm of the Germany Cystic Fibrosis Foundation Mukoviszidose e.V. (projects A02/08 and SIP CFTR-1-2009).

\section{References}

1 Bush A, Alton EWFW, Davies JC, Griesenbach U, Jaffe A(eds): Cystic Fibrosis in the 21st century. Basel, Karger, 2006.

2 Gentzsch M, Chang XB, Cui L, Wu Y, Ozols VV, Choudhury A, Pagano RE, Riordan JR: Endocytic trafficking routes of wild type and DeltaF508 cystic fibrosis transmembrane conductance regulator. Mol Biol Cell 2004;15:2684-2696.

3 Ward CL, Omura S, Kopito RR: Degradation of CFTR by the ubiquitin-proteasome pathway. Cell 1995;83:121-127.

4 Wang X, Venable J, LaPointe P, Hutt DM, Koulov AV, Coppinger J, Gurkan C, Kellner W, Matteson J, Plutner H, Riordan JR, Kelly JW, Yates JR 3rd, Balch WE: Hsp90 cochaperone Aha1 downregulation rescues misfolding of CFTR in cystic fibrosis. Cell 2006;127:803-815.

5 Denning GM, Anderson MP, Amara JF, Marshall J, Smith AE, Welsh MJ: Processing of mutant cystic fibrosis transmembrane conductance regulator is temperature-sensitive. Nature 1992;358:761-764.

6 Gentzsch M, Choudhury A, Chang XB, Pagano RE, Riordan JR: Misassembled mutant DeltaF508 CFTR in the distal secretory pathway alters cellular lipid trafficking. J Cell Sci 2007;120:447-455.

7 Cholon DM, O`Neal WK, Randell SH, Riordan JR, Gentzsch M: Modulation of endocytic trafficking and apical stability of CFTR in primary human airway epithelial cultures. Am J Physiol Lung Cell Mol Physiol 2010;298:L304-314. 
van Barneveld/Zander/Hyde et al.: Mutant CFTR in Lung

-8 Aleksandrov AA, Kota P, Aleksandrov LA, He L, Jensen T, Cui L, Gentzsch M, Dokholyan NV, Riordan JR: Regulatory insertion removal restores maturation, stability and function of DeltaF508 CFTR. J Mol Biol 2010;401:194-210.

$>9$ Serohijos AW, Hegedus T, Aleksandrov AA, He L, Cui L, Dokholyan NV, Riordan JR: Phenylalanine-508 mediates a cytoplasmic-membrane domain contact in the CFTR 3D structure crucial to assembly and channel function. Proc Natl Acad Sci USA 2008;105:3256-3261.

10 Gené GG, Llobet A, Larriba S, de Semir D, Martínez I, Escalada A, Solsona C, Casals T, Aran JM: Nterminal CFTR missense variants severely affect the behavior of the CFTR chloride channel. Hum Mutat 2008;29:738-749.

11 Kartner N, Augustinas O, Jensen TJ, Naismith AL, Riordan JR: Mislocalization of delta F508 CFTR in cystic fibrosis sweat gland. Nat Genet 1992;1:321-327.

12 Kälin N, Claass A, Sommer M, Puchelle E, Tümmler B: DeltaF508 CFTR protein expression in tissues from patients with cystic fibrosis. J Clin Invest 1999;103:1379-1389.

13 Mall M, Kreda SM, Mengos A, Jensen TJ, Hirtz S, Seydewitz HH, Yankaskas J, Kunzelmann K, Riordan JR, Boucher RC: The DeltaF508 mutation results in loss of CFTR function and mature protein in native human colon. Gastroenterology 2004;126:32-41.

14 van Barneveld A, Stanke F, Tamm S, Siebert B, Brandes G, Derichs N, Ballmann M, Junge S, Tümmler B: Functional analysis of F508del CFTR in native human colon. Biochim Biophys Acta 2010;1802:1062-1069.

15 Kreda SM, Mall M, Mengos A, Rochelle L, Yankaskas J, Riordan JR, Boucher R: Characterization of wildtype and deltaF508 cystic fibrosis transmembrane regulator in human respiratory epithelia. Mol Biol Cell 2005;16:2154-2167.

16 Amaral MD, Kunzelmann K: Molecular targeting of CFTR as a therapeutic approach to cystic fibrosis. Trends Pharmacol Sci 2007;28:334-341.

17 Accurso FJ, Rowe SM, Clancy JP, Boyle MP, Dunitz JM, Durie PR, Sagel SD, Hornick DB, Konstan MW, Donaldson SH, Moss RB, Pilewski JM, Rubenstein RC, Uluer AZ, Aitken ML, Freedman SD, Rose LM, MayerHamblett N, Dong Q, Zha J, Stone AJ, Olson ER, Ordoñez CL, Campbell PW, Ashlock MA, Ramsey BW: Effect of VX-770 in persons with cystic fibrosis and the G551D-CFTR mutation. N Engl J Med 2010;363:1991-2003.

18 Welsh MJ, Smith AE: Molecular mechanisms of CFTR chloride channel dysfunction in cystic fibrosis. Cell 1993;73:1251-1254.

19 Zielenski J: Genotype and phenotype in cystic fibrosis. Respiration 2000;67:117-133.

20 Bradford MM: A rapid and sensitive for the quantitation of microgram quantitites of protein utilizing the principle of protein-dye binding. Anal Biochem 1976;72:248-254.

21 Thiemann A, Gründer S, Pusch M, Jentsch TJ: A chloride channel widely expressed in epithelial and nonepithelial cells. Nature 1992;356:57-60.

-22 Lipecka J, Bali M, Thomas A, Fanen P, Edelman A, Fritsch J: Distribution of ClC-2 chloride channel in rat and human epithelial tissues. Am J Physiol Cell Physiol 2002;282:C805-816.

23 Dupuit F, Kälin N, Brézillon S, Hinnrasky J, Tümmler B, Puchelle E: CFTR and differentiation markers expression in non-CF and deltaF508 homozygous CF nasal epithelium. J Clin Invest 1995;96:1601-1611.

24 Penque D, Mendes F, Beck S, Farinha C, Pacheco P, Nogueira P, Lavinha J, Malhó R, Amaral MD: Cystic fibrosis F508del patients have apically localized CFTR in a reduced number of airway cells. Lab Invest 2000;80:857-868.

25 Gee HY, Noh SH, Tang BL, Kim KH, Lee MG. Rescue of $\Delta$ F508-CFTR trafficking via a GRASP-dependent unconventional secretion pathway. Cell 2011;146:746-760.

26 Mummery JL, Killey J, Linsdell P: Expression of the chloride channel CLC-K in human airway epithelial cells. -Can J Physiol Pharmacol 2005;83:1123-1128. 